die Notwendigkeit einer transkulturellen Betrachtung hinzuzufügen.

Führt man sich zudem die gesellschaftliche, kulturelle Mentalität sowie das spezifische, emotionale Zusammenspiel von Zivilgesellschaft und Politik in China vor Augen, darf man nicht die Angst auf beiden Seiten übersehen: Die Angst der Menschen vor einer zu (zu) mächtigen Politik, aber auch die Angst der Politik vor der Emotionalität der Menschen, vor der gesellschaftlich-kulturellen «AffektBereitschaft» in China. Insoweit scheinen Gesellschaft und Politik (auch) durch Angst miteinander verbunden, sowie voneinander abhängig zu sein. Dieses Beziehungsmuster scheint eine wechselseitige Stabilisierung zu bewirken. Deutet eine solche Beziehung gar auch auf ein im Verborgenen liegendes kulturelles Beziehungsmuster Chinas hin?

Unschulds Buch, seine kundigen und differenzierten Ausführungen über die kollektive Traumatisierung in China beleuchten einerseits die spezifische Situation in China und in Bezug auf die chinesische Gesellschaft. Andererseits verdeutlicht Unschuld die enorme Herausforderung an eine psychologische, aber auch gesellschaftskritische Betrachtung von kollektiver Traumatisierung generell. Ist es doch sinnvoll und wichtig, sich sehr genau mit der spezifischen Situation in einer Population, einer Gesellschaft oder einem kollektiven Raum zu befassen, bevor man (leider oftmals voreilig) Rückschlüsse zieht; Rückschlüsse darüber, wie man die Situation (leider oftmals nur von aussen gesehen) meint bewerten zu müssen. Hierauf aufbauend ist es ebenso wichtig, einen spezifischen Umgang mit einer solchen Situation abzuleiten.

Ist man als Psychotherapeut in diesem kollektiven Erfahrungsraum unterwegs oder will man sich diesbezüglich ein relevantes Verständnis aneignen, bedarf es unbedingt einer transkulturellen Betrachtung. Diese bezieht sich auch auf die realen Verhältnisse in der jeweiligen Population oder Gesellschaft, auf kulturelle Verhaltens- und Beziehungsmuster. Diese Betrachtung würdigt gerade hierdurch das (kollektive) Erleben der jeweiligen Population/Gesellschaft zum gegenwärtigen Zeitpunkt der Entwicklung. Sie bezieht sich aber auch auf das emotionale Echo, die Gegenübertragung desjenigen, der eine solche Betrachtung vornimmt. Ist man als Betrachter selbst doch immer auch Teil des Geschehens.

Ulrich Sollmann

\section{Ursula Wirtz (2018). Stirb und werde. Die Wandlungskraft traumatischer Erfahrungen}

\section{Ostfildern: Patmos. 384 Seiten. 39,00 EUR}

\author{
Psychotherapie-Wissenschaft 8 (2) 92-93 2018 \\ www.psychotherapie-wissenschaft.info \\ CC BY-NC-ND \\ https://doi.org/10.30820/8243.20
}

Ein reichhaltiges Buch. Ursula Wirtz, Psychoanalytikerin nach C. G. Jung hat ein mit rund 400 Seiten umfassendes Buch über psychische Traumata und ihre Behandlung vorgelegt. Man merkt es der promovierten Philosophin und Germanistin an, sie hat mit dem Thema jahrelange Erfahrung.

Ihr gemäss sind traumatische Erfahrungen Grenzerfahrungen, die zwar die Macht haben, die Betroffenen zu zerstören, aber ebenso können diese über sich hinauswachsen und ihr Trauma transzendieren. Die Autorin ermöglicht uns mit ihrem Werk, einen Blick in die spirituelle Dimension zu werfen, die die Betroffenen reifen und weiser werden lassen.

Das macht eben die Reichhaltigkeit aus, denn Ursula Wirtz führt uns nicht nur in klinische Fallgeschichten und ihre Behandlungen ein, sondern auch in die Welt der Mythen und des kollektiven Wissens, wie Menschen früher mit Traumata und dem Bösen umgegangen sind, sei es mit den Geschichten der indischen Göttin Kali oder der griechischen Figur der Medusa oder der antiken Hadesfahrt, sei es mit den Antworten der Philosophen wie etwa Leibnitz oder Kant. Auch im Roten Buch von C. G. Jung ist dieser Umgang mit dem malum beschrieben. Oder dann beschreibt sie, wie die Maler Breughel, Kokoschka oder Goya mit dem Tod in ihren Bilder umgehen. Auch das tibetanische und das ägyptische Totenbuch werden erwähnt, die die Nachtmeer- und Unterweltfahrt und die Wiederauferstehung zum Inhalt haben. Für die Lesenden sind diese Beispiele wichtig, da es bei Traumata ja gerade um die fehlende Symbolisierung von inneren Gefühls- und Empfindungszuständen geht. Der Blick in die kollektive Kultur mag dann tröstend sein und dem Individuum Halt inmitten überschwemmender Erfahrungen geben.

Ursula Wirtz hat mit vielen Experten und Betroffenen weltweit Kontakt gehabt, wie mit dem kambodschanischen Psychiater Chhim Sotheara, der sich um die Überlebenden in Phnom Penh kümmerte, oder mit Holocaustund Jugoslawienkrieg-Überlebenden. Ihre persönliche Überzeugung ist, dass bei der Therapie Überlegungen von existenziellen «Grunderfahrungen ohne ein vertieftes spirituelles Verständnis zu kurz greifen, denn ausgesprochen oder unausgesprochen ist die spirituelle Ebene des Menschseins in dieser Arbeit immer konstelliert» (S. 64). Das ist eine Aussage, die man nur unterstützen kann.

C. G. Jung, selbst ein Betroffener von Traumata, wird ausführlich beschrieben, insbesondere seine Verarbeitung der existenziellen Krise nach der Trennung von Sigmund Freud in seinem berühmten Roten Buch. Die totale Umkehr alles Vertrauten wird erlebt, nirgends findet man 
Halt, das Alte stirbt ab und das Neue ist noch nicht da. Was tun? Jung hat sich entschlossen, seine inneren $\mathrm{Zu}$ stände zu malen, daraus entstand schliesslich Das Rote $B u c h$, das vor einigen Jahren veröffentlicht worden ist.

Traumabetroffene können in dem, was sie erlebt haben, keinen Sinn sehen, sie stehen an einer Schwelle, in einem Zustand der Liminalität und müssen irgendwie zurückfinden. Die Holocaust-Betroffenen Nelly Sachs, Paul Celan, Primo Levi oder Rose Ausländer konnten ihre Erfahrungen poetisch umsetzen, was Ursula Wirtz in einem sehr berührenden Kapitel darstellt. Wie kann man seine Poesie inmitten des Grauens bewahren, wie kann man Sinn in rhythmischen Versen wiederfinden und die Paradoxien beschreiben, in Versen, die die traumatische Wunde transzendieren? Das alles beschreibt die Autorin mit einfühlenden Worten und tastet sich zu der Wandlungsenergie vor, die das Versöhnen, Verzeihen und Vergeben mit sich bringt und die doch so schwierig zu erreichen ist. Der Trauerprozess führt dazu, dass Ideale zerbrechen und dass Widersprüche bestehen bleiben können, auch mit seinem «Gott», dass man endlich ist, ohne daran zu zerbrechen. Das ist das, was die Wandlung mit sich bringt und was nur gelingt, wenn man seinen eigenen Schatten integriert. Hier vermisst man zuweilen die klare Aussage gegenüber den Tätern, die rücksichtlos und durchdrungen von Machtbesessenheit die Opfer missbrauchen. Die Traumaopfer suchen ja nicht selten den Fehler bei sich, um dem Ganzen einen Sinn abzugewinnen, und die Aggression in Form von eigener Meinung und klarer Haltung ist etwas, was sie oft noch lernen müssen.

Allerdings betont Wirtz immer wieder die weibliche Aggressionskraft, was in diesem Zusammenhang wichtig ist. Rund die Hälfte des Buches wird denn auch der Behandlung von Traumata gewidmet, in der die Jungianerin ausführlich auf die Phänomene und das Geschehen in der klinischen Praxis eingeht.

Im zweiten, klinischen Teil beschreibt Wirtz, wie hilfreich innere Bilder und Symbole sind, die im gemeinsamen imaginativen Raum in der Therapie auftauchen und die helfen, die traumatischen Erfahrungen einzuordnen und das chaotische traumatische Material zu reflektieren und neu zu bewerten. Sie beschreibt dabei, wie die therapeutische Beziehung unerlässlich ist, um diesen container herzustellen, ohne den ein spielerisches Erproben von neuen Lebensmöglichkeiten nicht möglich ist. Auch hier verweist sie des Öfteren auf Das Rote Buch von Jung, das die Möglichkeiten von Aktiver Imagination immer wieder aufzeigt. Sie verweist aber interdisziplinär auch auf andere Trauma-Methoden wie das EMDR (Eye Movement Desensitization and Reprocessing) oder auf die TRIMB(Trauma-Rekapitulation-Imagination-Motion-Breath)Methode, die in Deutschland in einer Klinik für komplex traumatisierte Patienten entwickelt wurde. Bilder von Zeichnungen oder Gestaltungen ihrer Klienten werden ebenfalls aufgeführt, wie auch Texte von ihnen.

Ganz wichtig ist Wirtz der Aspekt der Komplementarität, ohne den das Trauma nicht verstanden werden kann. Trauma und Spiritualität lassen sich unter diesem Aspekt verstehen, Ich-Stärkung und Heilung setzt sie in Bezug zu Ich-Transzendenz und Heil, Sprechen zum Schweigen, Dialog zur Meditation, Reflexion zur reinen Präsenz. Komplementarität meint eine Haltung des «Sowohl-als-auch» im Unterschied zum «Entweder-oder». Als Jungianerin vergisst sie die Transzendente Funktion nicht, die im Hin und Her der verschiedenen Argumente das Dritte finden lässt und die von C. G. Jung als Prozess bereits 1916 beschrieben wurde. Das Gegenteil lässt sich oft umdrehen, sodass man wieder in Kontakt mit den schöpferischen Kräften kommen kann. Oder in ihren Worten: «Wenn sich ein Mensch festgefahren oder durch traumatischen Rückzug in sich selbst eingekapselt fühlt, dann bringt die Energie der Transzendenten Funktion als prozessorientierte Energie wieder Bewegung in die Psyche, und die Lebensenergie kann sich neuen Zielen zuwenden» (S. 217). Die Spannung der Gegensätze ermöglicht neues Wachstum. Dafür muss man falsche Einstellungen opfern. Das Opfermotiv spielt eine wichtige Rolle in diesem Kapitel, in Trauerprozessen muss jeweils etwas geopfert werden. Hier verweist Wirtz auch auf kollektives Wissen, wie es etwa in Märchen und Mythen vorkommt. Man würde in diesen Kapiteln gerne mehr vom Jungianer Donald Kalsched wissen, der ausführlich die archetypische Abwehr bei traumatisierten Menschen beschrieben hat.

Alles in allem ist das Buch von Ursula Wirtz ein wirklich reichhaltiges Buch, es zeugt von jahrelanger Erfahrung mit dem Thema, es zeugt aber auch von einem fundierten Wissen, wie sich diese Prozesse in Mythen, Geschichten, Philosophie, in Kultur überhaupt widerspiegeln.

Isabelle Meier 